\title{
Über die Gültigkeit des Alles oder Nichts=Gesetzes für die Kontraktion des Schweineureters.
}

\author{
Von \\ Masatake Yano. \\ (矢野正武) \\ (Aus dem II. Institut der Physiologie der Tohoku \\ Reichsuniversität zu Sendai, \\ Vorstand: Prof. Dr. K. Motokazua.)
}

Einleitung.

Die Gültigkeit des Alles oder Nichts-Gesetzes ist bei glatten Muskeln nicht so genau wie bei Skelettmuskeln untersucht worden. Barbera $a^{1)}$ und Woodworthe gaben an, dass der glatte Muskel des Froschmagens nicht dem Alles oder Nichts-Gesetz folgt. Schülle $\mathrm{r}^{3)}$ sagte auch, dass der glatte Muskel des Mastdarms auf Extrareizung nicht immer gemäss diesem Gesetz antwortet. In letzter Zeit hat Aiz a wa ${ }^{4}$ gefunden, dass wenn der Schweineureter elektrisch an einem Punkte des Nierenendes, Mittelteils oder Blasenendes gereizt wird, er die Alles oder Nichts-Kontraktion ausfürt. Aber die Abstufung der Reizstärke wurde bei seinem Versuch nicht mit genügender Genauigkeit ausgeführt, so dass die Frage noch offen bleibt, ob der Ureter auch bei noch genauerer Schwelleneingrenzung streng dem Alles oder Nichts-Gesetz folgt oder nicht.

\section{Methode.}

Wie in Fig. 1 gezeigt ist, befand sich ein Glasgefäss (etwa $15 \mathrm{~cm}$ lang, $5 \mathrm{~cm}$ breit, $4 \mathrm{~cm}$ tief)in einem anderen, das als Thermostat diente, dessen Temperatur bei diesen Untersuchungen auf 18,28 oder $38^{\circ} \mathrm{C}$ beibehalten wurde. Der ausgeschnittene Schweineureter lag, immer mit Luftbläschen versorgt, in der Ty rodeschen Lösung des inneren Glasgefässes. Man reizte den Ureter an einem Punkte des Nierenendes, Mittelteils oder Blasenendes mit Gleichstrom aus Bleiakkumulatoren und zeichnete die Längs- oder Zirkulärkontraktionen (bei Doppelreizung nur die letzteren) der oben gennanten drei Punkte auf das 
Fig. 1. (A) Versuchsanordnung.

(B) Methode zur Registrierung der Längskontraktion als ganzes.

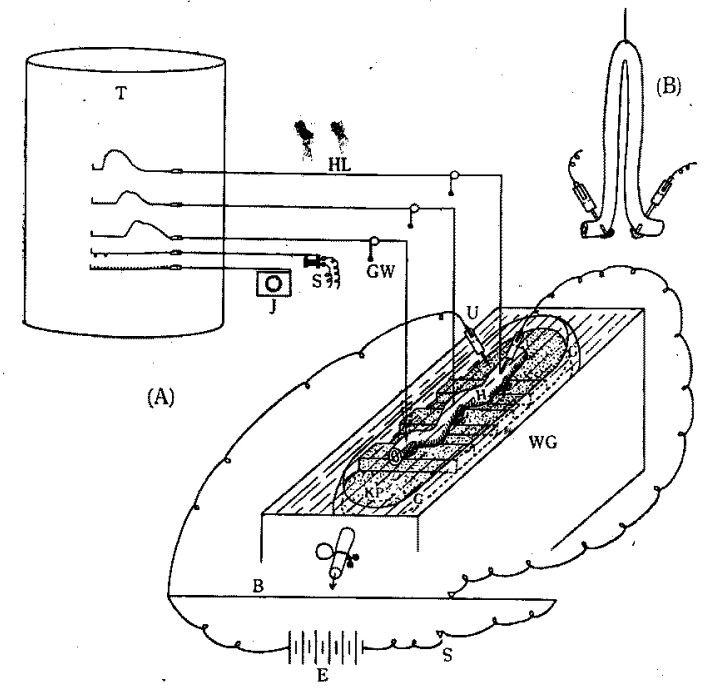
B. Brückendraht.
E. Batterie.
G. Glasgefäss.
GW. Gewicht.
H. Harnleiter.
HL. Hebel.
J. J a quetsches Chronometer.
KP. Korkplatte.
S. Schlüssel.
T. Trommel.
U. Unpolarisierbare Fadenelektrode.
WG. Wassergefäss.

Russpapier des Kymographions gleichzeitig auf. Die Kontraktionen wurden durch Hebel 6 fach vergrössert, an denen ein Gewicht von $20 \mathrm{~g}$ gehängt war, so dass die wirkliche Belastung für den Ureter 2,5 $\mathrm{g}$ betrug.

Der Reizstrom wurde von 6 Akkumulatoren von $2 \mathrm{~V}$ (E in Fig. 1) gespeist, die an beiden Enden eines Brückendrahtes $(100 \mathrm{~cm}$ lang, B in Fig. 1) verbunden waren, und durch unpolarisierbare Gipselektroden dem Ureter zugeleitet. Die Reizdauer betrug mehrere Sekunden bei Einzelreizung und eine halbe Sekunde bei Doppelreizung, unter Umständen aber wurde sie nur im ersten Fall unbegrenzt lang gewählt. Die Anode befand sich in der Flüssigkeit, die Kathode war aber auf dem zu reizenden Ort aufgesetzt. Um der Stromschleife vorzubeugen, liess man nur bei der Reizung die Flüssigkeit um den Ureter etwas herausfliessen, so dass der Reizpunkt des Ureters zum Teil, etwa einen halben Millimeter hoch blossgelegt wurde.

Bei der Schwellenbestimmung wurde die Reizspannung um $1 \mathrm{~mm}$ $(0,012 \mathrm{~V})$ des Brückendrahtes bei Einzelreizung reguliert, aber bei 
Doppelreizung war die Schwelleneingrenzung etwas gröber; sie entsprach nämlich der Genauigkeit von $5 \mathrm{~mm}$ bis $5 \mathrm{~cm}$ des Brückendrahtes. Bei der Bestimmung des Refraktärstadiums wurde der Reiz um $10 \mathrm{~cm}$ des Brückendrahtes verstärkt, wenn die zweite Reizung unwirksam blieb. Auf diese Weise verfolgte ich das kleinste Reizintervall, wo die zweite maximale Reizung (12 V) eben unwirksam war.

\section{Ergebnisse.}

I. Refrakärstadium.

Im allgemeinen ist das absolute Refraktärstadium ein wenig länger als die Verkürzungszeit der Kontraktion an der gereizten Stelle.

Fig. 2. Erregbarkeitskurve bei verschiedenen Temperaturen. $A, B, C$ : Bezeichnung der Präparate.

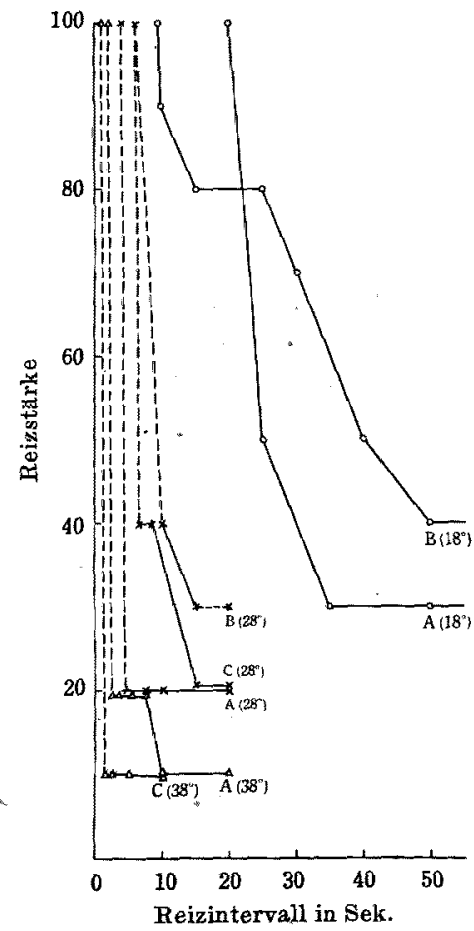

Das absolute Refraktärstadium beträgt bei $18^{\circ} \mathrm{C} 12,5-15,5$ Sekunden, bei $28^{\circ} \mathrm{C} 3,5-6,5$ Sek. und bei $38^{\circ} \mathrm{C}$ 1,5-3,0 Sek. Nach Aiza w a beträgt dasselbe $2-3$ Sek. bei $38^{\circ} \mathrm{C}$. Der Temperaturquotient ist also 2-3. Bei $18^{\circ} \mathrm{C}$ kommt es vor, wenn auch selten, dass das Refraktärstadium kürzer als die Verkürzungszeit der ersten Kontraktion ist.

Die Erregbarkeitskurve verläuft natürlich desto steiler, je höher die Temperatur ist. (Fig. 2).

II. Eigenschaften der Kontraktion.

(a) Kontraktionshöhe.

Die Kontraktionshöhe bleibt, abgesehen von der allmählichen Veränderung oder Ermüdung, sowohl bei direkter als auch bei fortgeleiteter Erregung stets gleich, folgt also dem Alles oder Nichts-Gesetz.

Die Kontraktionshöhe ist verschieden je nach der Stelle des Ureters. Sie ist in den meisten Fällen am Nierenende grösser als am Blasenende, wenn es sich um die Zirkulärkontraktion handelt, das Verhältnis ist aber bei der Längskontraktion gerade umgekehrt. 
Diese Tatsache ist darauf zurückzuführen, dass derZirkulärmuskel am Nierenende, der Längsmuskel aber am Blasenende am besten entwickelt ist. Die Höhe der Längrs- oder Zirkulärkontraktion nimmt am Mittelteil des Ureters in der Mehrzahl der Fälle auch die Mittelstellung ein, wie Aiza wa darauf hinweist.

Schultz's), Eckstein ${ }^{6}$ (beide, Froschmagen) und Stewart ${ }^{3)}$ (Katzenblase) bemerkten, dass sich die Kontraktionshöhe mit der Temperatursteigerung vergrössert. Nach Schultz und Stewart soll sich aber die Kontraktion über $39^{\circ}-40^{\circ} \mathrm{C}$ verkleinern.

Bei meiner Untersuchung ist die Kontraktionshöheim allgemeinen bei niedrigeren Temperaturen grösser als bei höheren, aber sie kann unter Umständen von der Temperatur fast unabhängig konstant bleiben, oder allmählich mit der Temperatursteigerung zunehmen. Diese Neigung ist am Blasenende häufiger zu bemerken.

Die Kontraktionshöhe ist in Tabelle I zusammengestellt.

Tabelle I.

Die Kontraktionshöhe des Vreters.

\begin{tabular}{c|c|c|c|c}
\hline & $\begin{array}{c}\text { Temperatur } \\
\text { inc }\end{array}$ & $\begin{array}{c}\text { Grösste } \\
\mathrm{mm}\end{array}$ & $\begin{array}{c}\text { Kleinste } \\
\mathrm{mm}\end{array}$ & $\begin{array}{c}\text { Durch schnitt } \\
\mathrm{mm}\end{array}$ \\
\hline \multirow{2}{*}{ Längs- } & 18 & 6,5 & 2,8 & 3,6 \\
kontraktion & 28 & 5,8 & 1,4 & 2,6 \\
& 38 & 2,3 & 0,33 & 0,9 \\
\hline \multirow{2}{*}{ Zirkulär- } & 18 & 4,7 & 0,36 & 2,07 \\
kontraktion & 28 & 4,1 & 0,33 & 1,8 \\
& 38 & 4,4 & 0,08 & 1,67
\end{tabular}

(b) Latenz.

Steinhause ${ }^{8)}$ bestätigte, dass die Latenzzeit beim Sartorius rasch zunimmt, wenn sich der Reiz dem Schwellenwert nähert; nach diesem Autor besteht eine Hyperbelbeziehung zwischen Latenz und Reizstärke.

Im Gegensatz dazu bleibt die Latenz der Kontraktion in meinen Versuchen bei gleicher Temperatur stets konstant, sei es nun der Schwellenreiz oder der maximale Reiz.

Schultz (Froschmagen, $-5^{\circ} \mathrm{C}-0^{\circ}-50^{\circ} \mathrm{C}$ ) gibt an, dass sich die Latenz mit der Temperatursteigerung verkleinert, aber über $39^{\circ} \mathrm{C}$ wieder zunimmt.

Die Abhängigkeit der Latenzzeit von der Temperatur ist in Tab. II dargestellt. 
Tabelle II. Latenz. (Zirkulärkontraktion)

\begin{tabular}{c|c|c|c}
\hline $\begin{array}{c}\text { Temperatur } \\
\text { in }{ }^{\circ} \mathrm{C}\end{array}$ & Grösste & Kleinste & $\begin{array}{c}\text { Durch- } \\
\text { sehnitt }\end{array}$ \\
\hline 18 & $2,0^{\prime \prime}$ & $1,5^{\prime \prime}$ & $1,7^{\prime \prime}$ \\
28 & $1,0^{\prime \prime}$ & $0,7^{\prime \prime}$ & $0,8^{\prime \prime}$ \\
38 & $0,5^{\prime \prime}$ & $0,4^{\prime \prime}$ & $0,4^{\prime \prime}$ \\
43 & - & - & $0,5^{\prime \prime}$
\end{tabular}

Die Latenz der Längskontraktion ist etwas länger als die der Zirkulärkontraktion und der Temperaturquotient der Latenz ist 2 , wie aus Tab.II ohne weiteres hervorgeht.

Die Latenz verlängert sich natürlich bei Ermüdung. In dieser Hinsicht ist besonders hervorzuheben, dass die Ermüdung oft ausgeprägter in dieser Verlängerung derLatenz ihren Ausdruck findet als in der Abnahme der Kontraktionshöhe; die Latenz kann sich merklich verlängern, während die Kontraktion noch gleich gross bleibt.

(c) Verkürzungszeit.

Die Verkürzungszeit der Kontraktion bleibt entsprechend ihrer Alles oder Nichts-Kontraktion bei aller Reizungsstärke konstant. Sie

Tabelle III.

Die Verkürzungszeit der Zirkulärkontraktion.

\begin{tabular}{c|c|c|c}
\hline $\begin{array}{c}\text { Temperatur } \\
\text { in }{ }^{\circ} \mathrm{C}\end{array}$ & Grösste & Kleinste & $\begin{array}{c}\text { Durch- } \\
\text { sehnitt }\end{array}$ \\
\hline 18 & $17,0^{\prime \prime}$ & $6,8^{\prime \prime}$ & $11,7^{\prime \prime}$ \\
28 & $6,0^{\prime \prime}$ & $2,0^{\prime \prime}$ & $3,8^{\prime \prime}$ \\
38 & $3,5^{\prime \prime}$ & $0,8^{\prime \prime}$ & $1,8^{\prime \prime}$
\end{tabular}

Nach Stewart (Katzenblase, $10^{\circ}-55^{\circ} \mathrm{C}$ ) ist die Verkürzungszeit um so kürzer, je höher die Temperatur ist. Schultz (Froschmagen) gab an, dass sich die Kontraktionsdauer mit der Temperatursteigerung von $5^{\circ}$ bis $42,5^{\circ} \mathrm{C}$ immer verkleinert, aber von $42,5^{\circ}$ bis $48^{\circ} \mathrm{C}$ demgegenüber allmählich verlängert (nach seinem Schema).

Die Form des aufsteigenden Schenkels der Kontraktionskurve ist auch beachtenswert; die Kurve auf direkte Reizung hin steigt von der Grundlinie sofort steil an, während ist in Tabelle III zusammengestellt.

Die Längskontraktion ist im Durchschnitt etwas länger als bei der Zirkulärkontraktion. Ihr Temperaturquotient ist $2-3$, wie sich aus der obigen Tabelle ohne weiteres ergibt.

Fig. 3. Unterschied in Kurvenform zwischen fortgeleitieten und durch direkten Reiz hervor. gerufenen Kontraktionen (Längskontr.)

a: Kurven am Reizort.

b: fortgeleitete Kontraktionen.

a
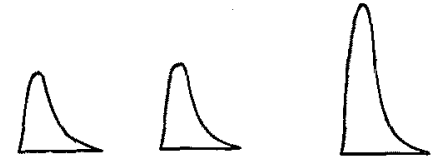

b
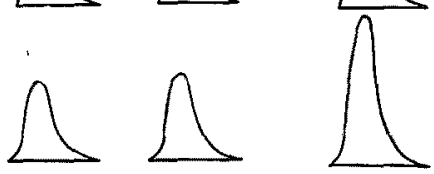

Nierenende Mittelteil Blasenende 
diejenige durch fortgeleitete Erregung anfangs langsam (während 1-2 Sek.), dann steiler aufsteigt (Fig. 3). Daher kann die Verkürzungszeit je nach der Form etwas verschieden sein.

\section{(d) Leitungsgeschwindigkeit.}

Die Leitungsgeschwindigkeit der Kontraktion vom Nierenende nach dem Mittelteil hin sowie von diesem nach dem Blasenende hin ist meist ein wenig grösser als die in der entgegengesetzten Richtung, wenn dieser Unterschied auch nicht so merklich ist.

Im Durchschnitt hat die

Tabelle IV.

\begin{tabular}{c|c|c|c}
\hline \multirow{2}{*}{$\begin{array}{c}\text { Temperatur } \\
\text { in }{ }^{\circ} \mathrm{C}\end{array}$} & \multicolumn{3}{|c}{$\begin{array}{c}\text { Leitungsgeschwindigkeit } \\
\text { mm/Sek. }\end{array}$} \\
\cline { 2 - 4 } & Grösste & Kleinste & $\begin{array}{c}\text { Durch- } \\
\text { schnitt }\end{array}$ \\
\hline 18 & 18 & 5 & 10,2 \\
28 & 30 & 9 & 18,1 \\
38 & 35 & 13 & 24,4
\end{tabular}

Leitungsgeschwindigkeit folgenden Wert.

Nach Engelmann ${ }^{9}$ verringern alle Einflüsse, welche die Reizbarkeit des Ureters herabsetzen, wie $\mathbf{z}$. B. Abkühlung, Entziehung der Blutzufuhr, Ermüdung usw. auch sein Leitungsvermögen. Diese Verringerung zeigt sich zunächst darin, dass die Kontraktionswelle, je weiter sie sich von ihrem Ausgangspunkt fortpflanzt, um so schwächer wird, um endlich zugrundezugehen.

Dies Verhältnis wurde auch in meinen Versuchen bestätigt; je nach dem Ermüdungsgrad verlängert sich die Leitungszeit in verschiedenem Masse, dann geht das Leitungsvermögen in der Mitte ganz verloren und bei hochgradiger Ermüdung reagiert der Ureter nur noch am Reizpunkt. Merkwürdigerweise zeigt in diesem ermüdeten Zustand der Ureter doch die Alles oder Nichts-Kontraktion; die Kontraktion ist wohl dabei klein oder auf ein Lokal beschränkt, aber ihre Grösse bleibt doch von der Reizstärke unabhängig ganz konstant.

(e) Über die Kontraktion des Ureters als ganzes.

Die Wirkung der Anode sowie Kathode auf den glatten Muskel ist schon von einigen Autoren studiert worden. Nach Lüderitz ${ }^{10)}$ (Katze, Kaninchen, Meerschweinchen, Darm in situ) ist die Kathodenwirkung stärker als die Anodenwirkung. Biedermann ${ }^{11)}$ (Hund, Kaninchen, Meerschweinchen, Ureter in situ) bestätigte, dass sich der Ureter bei der Schliessung des Stromkreises stets zuerst an der Anode zusammenschnürte. Schillbach ${ }^{12)}$ erblickte bei der Schliessung, dass an der Anode peristaltische Wellen besonders in aufsteigender 
Richtung hervortreten, an der Kathode dagegen meist Ene lokale Kontraktion auftritt.

Ich legte die Elektroden auf zwei beliebige Teile der folgenden drei Abschnitte des Ureters, nämlich Nierenende, Mittelteil und Blasen-.. ende und untersuchte die Reizwirkung der einzèlnen Pole, wobei die Reizdauer 2 Sekunden betrug. Wenn der Reiz etwas ubetsuwellig ist, tritt die Kontraktion in den meisten Fällen nur an der Kathode, und vereinzelt nur an der Anode auf. Verstärkt man aber den Reiz über einen gewissen Grad, so treten die Kontraktionen an beiden Polen gleichzeitig auf. Wie in Tabelle V, A gezeigt ist, treten die Kontraktionen bei $7,452 \mathrm{~V}$ sowohT an der Kathode (Blasenende) als auch an der Anode(Nierenende) gleichzeitig auf und pflanzen sich nach dem Mittelteil hin fort. Unter Umständen tritt die Kontraktion nur an einem Pol auf, wie stark auch der Reiz sein mag (s. Tab. V, B).

Um zu sehen, welcher Unterschied in der Kontraktion des Ureters als ganzes zwischen den oben erwähnten zwei Formen der Erregung besteht, wurde folgende Untersuchung angestellt. Ich befestigte das Nieren- und Blasenende und hängte den Mittelteil an den Hebel, um die Zusammenziehung des Ureters als ganzeszu registrieren, wobei die

\author{
Tabelle V. \\ Erregung bei Kathode und Anode.
}

(A) Gleichzeitiges Auftreten der Kontraktion an beiden Polen (ausgezogene Pfeile). Gestrichelte Pfeile zeigen fortgeleitete Wellen.

\begin{tabular}{|c|c|c|c|c|c|}
\hline $\begin{array}{c}\text { Reizstarare } \\
\text { (V) }\end{array}$ & $\mid \begin{array}{c}\text { Nierenende } \\
\text { Anode } \\
\text { Kontraktions- } \\
\text { höhe in mm }\end{array}$ & $\begin{array}{c}\text { Richtung der } \\
\text { Leitung }\end{array}$ & $\begin{array}{c}\text { Mittelteil } \\
\text { Kontraktions- } \\
\text { höhe in mm }\end{array}$ & $\begin{array}{c}\text { Richtung der } \\
\text { Leitung }\end{array}$ & $\begin{array}{c}\text { Blasenende } \\
\text { Kathode } \\
\text { Kontraktions- } \\
\text { höhe in mm }\end{array}$ \\
\hline 3,6 & 4,0 & $\leftarrow \ldots$ & 3,5 & $\leftarrow$ & 7,8 \\
\hline 7,2 & 4,0 & $\leftarrow---$ & 3,5 & $\leftarrow$ & 7,9 \\
\hline 12,0 & 4,0 & $\longrightarrow$ & 3,6 & $\leftarrow$ & 7,8 \\
\hline 7,8 & 4,2 & 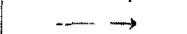 & 3,6 & 4 & 8,1 \\
\hline 7,4 & 4,4 & $\leftarrow-\cdots$ & 3,7 & $\leftarrow$ & 8,1 \\
\hline 7,46 & 4,2 & $\longrightarrow$ & 3,8 & $\leftarrow$ & 8,2 \\
\hline 7,452 & 4,4 & $\longrightarrow$ & 3,9 & $\leftarrow$ & 8,3 \\
\hline 7,4 & 4,4 & $\leftarrow---$ & 3,9 & $\leftarrow$ & 8,4 \\
\hline
\end{tabular}

(B) Auftreten der Kontraktion nur bei der Kathode.

\begin{tabular}{|c|c|c|c|c|c|}
\hline$(\mathrm{V})$ & $\begin{array}{l}\text { Nierenende } \\
\text { mm }\end{array}$ & $\begin{array}{l}\text { Richtung der } \\
\text { Leitung }\end{array}$ & $\begin{array}{c}\text { Mittelteil } \\
\text { Kathode } \\
\text { mm }\end{array}$ & $\begin{array}{l}\text { Richtung der } \\
\text { Leitung }\end{array}$ & $\begin{array}{c}\text { Blasenende } \\
\text { Anode } \\
\text { mm }\end{array}$ \\
\hline $\begin{array}{c}2,4 \\
3,0 \\
2,412 \\
12,0\end{array}$ & $\begin{array}{l}0 \\
2,8 \\
2,8 \\
2,7\end{array}$ & $\leftarrow$ & $\begin{array}{l}0 \\
3,4 \\
3,4 \\
3,3\end{array}$ & $\underset{\longrightarrow}{\longrightarrow} \rightarrow$ & $\begin{array}{l}0 \\
4,8 \\
4,7 \\
4,8\end{array}$ \\
\hline
\end{tabular}


Reizelektroden auf die befestigten Teile aufgesetzt wurden (Fig. 1, B). Wie eingangs erwähnt, liess ich die Tyrodesche Lösung während der Reizung ganz ausfliessen. Die auf diese Weise registrierte Kurve

Tabelle VI.

Abhängigkeit der Kontraktion des Ureters als ganzes von der Reizstärke.

(A) Abhängigkeit von der Reizstärke.

\begin{tabular}{r|c|c}
\hline $\mathrm{V}$ & $\begin{array}{c}\text { Kontraktions- } \\
\text { höhe in mm }\end{array}$ & $\begin{array}{c}\text { Verkürzungs- } \\
\text { zeit in Sek. }\end{array}$ \\
\hline 4,2 & 2,2 & 20 \\
8,4 & 4,3 & 21,5 \\
12,0 & 5,0 & 25
\end{tabular}

(B) Unabhängig keit von der Reizstärke.

\begin{tabular}{r|l|l}
\hline 12,0 & 7,7 & 33 \\
7,2 & 7,6 & 38 \\
3,6 & 7,9 & 38,5 \\
2,4 & 0 & 0 \\
3,0 & 7,7 & 40,5
\end{tabular}
blieb bei einigen Präparaten ganz unabhängig von der Reizstärke konstant, vergrösserte sich aber bei anderen Präparaten mit der Zunahme der Reizstärke (s. Tab. VI u. Fig. 4). Diese letztere Tatsache ist besonders beachtenswert, denn sie könnte zum Irrtum führen, dass die Kontraktion des Ureters nicht dem Alles oder Nichts-Gesetz folge. Nach meiner Ansicht beruht diese Tatsache auf der Summation der von den beiden Polen ausgegangenen Kontraktionen, aber nicht auf der Ungültigkeit des Alles oder Nichts-Gesetzes.

Fig. 4. Das scheinbare Versagen des Alles oder Nichts-Gesetzes bei der Längskontraktion des ganzen Ureters, untersucht mit der Methode in Fig. 1, B.



III. Kontraktion bei doppelter Reizung.

(a) Höhe der zweiten Kontraktion.

Die Höhe der durch die zweite Reizung hervorgerufenen Kontraktion ist je nach dem Reizintervall verschieden, und zwar desto kleiner, je kürzer das Reizintervall ist. Aber die Höhe der zweiten Kontraktion bleibt bei gegebenem Reizintervall ungeachtet der Reizstärke gleich hoch (s. Fig. 5).

Die Erholung der Kontraktilität nach dem absoluten Refraktärstadium geschiet um so rascher, je höher die Temperatur ist (s. Fig. 6). 
Fig. 5. Abhängigkeit der Kontraktionshöhe und Latenz rom Reizintervall.

K.H. Kontraktionshöhe.

L. Latenzzeit.

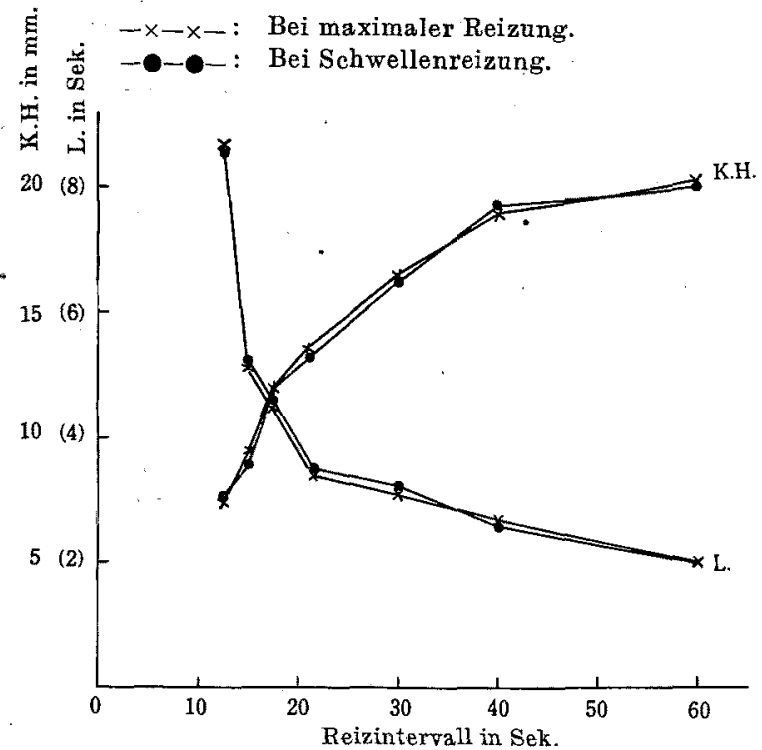

Fig. 6. Erholungskurve der Kontraktilität bei verschiedenen Temperaturen.

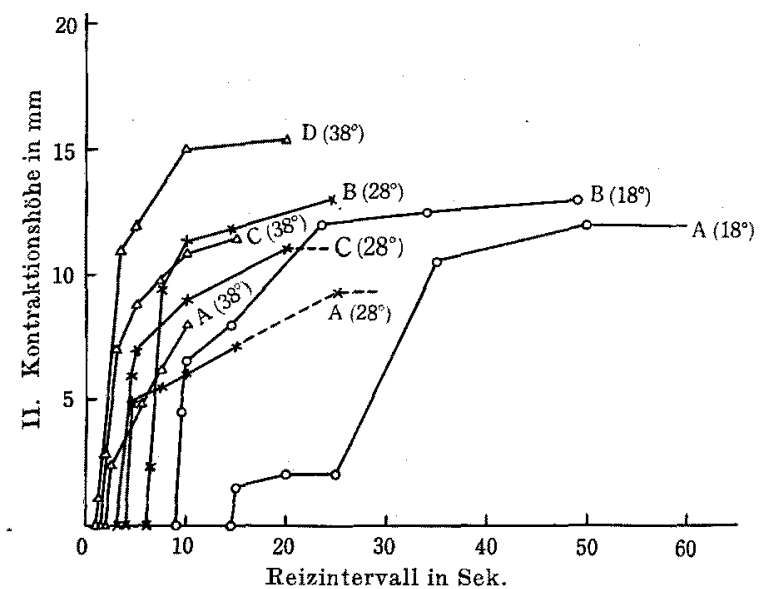

(b) Latenzzeit.

Beth $\mathrm{e}^{13)}$ befasste sich mit der Latenz der zweiten Kontraktion beim Skelettmuskel: Bei Doppelreizuug des Froschmuskels erweist 
sich die Latenz der zweiten Zuckung verlängert, wenn der zweite Reiz auf der ersten Zuckung oder in der ersten Hälfte ihrer Dekreszente einsetzt. Am Ende der Dekreszente und noch einige Zeit nach Ablauf der ersten Zuckung ist die Latenz gegenüber derjenigen des ausgeruhten Muskels verkürzt.

In meinem Falle ist die Latenz der zweiten Kontraktion desto grösser, je kürzer das Reizintervall ist. Die Neigung dieser Vergrösserung der Latenz ist um so steiler, je niedriger die Temperatur ist (s. Fig. 7), mit anderen Worten: die Erholung der Latenz ist um so rascher, je höher die Temperatur ist.

Fig. 7. Erholungskurve der Latenzzeit.

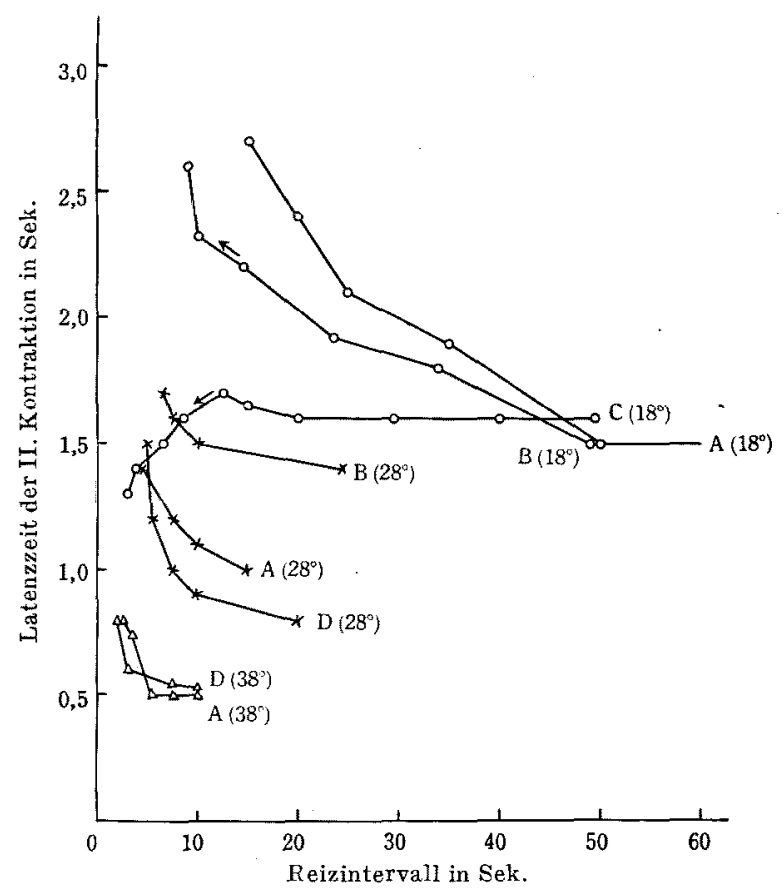

Wird das Reizintervall kürzer als die Verkürzungszeit bei $18^{\circ} \mathrm{C}$ gewählt, so ist die Summationskurve nicht mehr zweigipfelig, so dass die Analyse etwas schwieriger ist als bei der zweigipfeligen Kurve. In solchem Fall liess sich eine vorläufig unbegreifliche Erscheinung vereinzelt bemerken, nümlich dass die Latenz mit der Verkürzung des Reizintervalls statt Verlängerung sogar eine Verkürzung erfährt. Ob dies Verhalten auf irgendeinem Fehler in der Analyse oder auf anderen 
Umständen beruht, ist vorläufig nicht zu entscheiden (Fig. 7, C).

Wie aus Fig. 5 ohne weiteres hervorgeht, bleibt die Latenz der zweiten Kontraktion von der Reizstärke unabhängig, was darauf hinweist, dass die Latenzzeit bei doppelter Reizung auch dem' Alles oder Nichts-Gesetz folgt.

(c) Verkürzungszeit.

Die Verkürzungszeit nimmt umgekehrt zu ihrer Kontraktionsgrösse mit der Verkürzung des Reizintervalls zu. Sie verkleinert sich aber, wenn die zweite Kontraktion vor dem Gipfel der ersten Kontraktion entsteht, wie es selten bei $18^{\circ} \mathrm{C}$ vorkommt.

Die Erholungskurve ist ähnlich wie bei der Latenz.

(d) Leitungsgeschwindigkeit.

Die Leitungsgeschwindigkeit der zweiten Kontraktion ist um so kleiner bei je kürzerem Reizintervall. Der Abstand der beiden Kontraktionen wird nämlich mit der Fortleitung immer grösser, so dass die summierte Kurve mit der Fortleitung zweigipfelig wird, wie schon Aizawa darauf hinwies. Die Erholungskurve ist ähnlich derselben der Kontraktilität.

\section{Diskussion der Ergebnisse.}

Engelmann (Kaninchenureter, in situ) fand, dass sich am gereizten Orte die Kontraktionshöhe und -dauer mit der Zunahme der Reizstärke vergrössern, und dass sich die Latenz dabei verkleinert. Dasselbe Resultat erhielt $\mathrm{A} \mathrm{iba}^{14)}$ mit derselben Methodik wie En gel man n.

Dieser Befund, welcher dem Alles oder Nichts-Gesetz zu widersprechen scheint, ist ohne Schwierigkeit zu erklären, wenn man annimmt, dass die Erregung mit der Zunahme der Reizstärke an verschiedenen Punkten gleichzeitig auftritt. Meine obige Untersuchung weist darauf hin, dass dies wirklich der Fall ist. Die Alles oder Nichts-Kontraktion des Ureters ist nur dann zu erzielen, wenn die Dichte des Reizstroms an einem Reizpunkt eine genügende Grösse erreicht, um die Erregung nur aus diesem Punkt entstehen zu lassen. Handelt es sich um die fortgeleitete Erregung, so erhielten Engelmann und Aiba alle übereinstimmend die Alles oder Nichts-Kontraktion. Engelmann bemerkte, dass die unternormale Kontraktionswelle am Reizort in physiologischem Zustand des Ureters mit der Fortpflanzung an Stärke zunimmt und endlich die für einzelne Orte charakteristische maximale Grösse erreicht. Nach diesem Autor genügt dazu bereits die Fortpllanzung von etwa einigen Millimetern. Meine Resultate stimmen in 
bezug auf die fortgeleitete Erregung mit denjenigen Engelmanns und anderen völlig überein.

\section{Zusammenfassung.}

1. Das absolute Refraktärstadium des Ureters beträgt bei $18^{\circ} \mathrm{C}$ $12,5-15,5$, bei $28^{\circ} \mathrm{C} 3,5-6,5$ und bei $38^{\circ} \mathrm{C} 1,5-3,0$ Sek.

2. In bezug auf die Kontraktionshöhe und Latenzzeit folgt der Ureter dem Alles oder Nichts-Gesetz, welches in allen Zuständen des Präparates, z.B. im relativen Refraktärstadium, Ermüdung usw. seine Gültigkeit beibehält. Auch die Verkürzungszeit ist von der Reizstärke unabhängig.

3. Die Erholung der Kontraktilität, Latenz, Verkürzungszeit und Leitungsgeschwindigkeit ist um so rascher, je höher die Temperatur ist.

Es sei mir erlaubt, am Schluss Herren Ehrenprof. Dr. T. Fujita und Prof. Dr. K. Motokawa für ihre freundliche Leitung und Unterstützung meinen herzlichsten Dank auszusprechen.

\section{Literatur.}

(1) Barbera, A. G., Ztschr. f. Biologie, 1898, 36, 249

(2) Woodworth, R. S., Amer. J. Physiol., 1899-1900, 3, 44.

(3) Schüller, J., Arch. exp. Path. u. Pharmak., 1921, 9, 241.

(4) A izawa, S., Tohoku Ig, Z., 1936, 19, 33-38.

(5) Schultz, P., Arch. f. Physiol., 1897, 19.

(6) Eckstein, A., Pfügers Arch., 1920, 183, 63.

(7) Stew art, C. C., Amer. J. Physiol., 1901, 4, 202.

(8) Steinhausen, W., Pflügers Arch., 1921, 187, 35.

(9) Engelmann, Th. W., Pflügers Arch., 1869, 2, 265; 1870, 3, 248-289.

(10) L ü deritz, C., Pfügers Arch., 1891, 48, 9.

(11) Bied erm a n n, W., Pflügers Arch., 1889, 45, 386.

(12) Schillbach, E., Virchows Arch., 1887, 109, 278.

(13) Bethe, A., Pfügers Arch., 1937, 239, 19.

(14) A i ba, T., Nissin Igaku, 1934, 23, 2686. 